\title{
Evaluation of the immunogenicity of Campylobacter jejuni CjaA protein delivered by Salmonella enterica sv. Typhimurium strain with regulated delayed attenuation in chickens
}

\author{
Paweł Laniewski • Maciej Kuczkowski • Klaudia Chrząstek • \\ Anna Woźniak • Agnieszka Wyszyńska • Alina Wieliczko • \\ Elżbieta Katarzyna Jagusztyn-Krynicka
}

Received: 24 May 2013/Accepted: 19 July 2013/Published online: 4 August 2013

(C) The Author(s) 2013. This article is published with open access at Springerlink.com

\begin{abstract}
Campylobacter spp. are regarded as the most common bacterial cause of gastroenteritis worldwide, and consumption of chicken meat contaminated by Campylobacter is considered to be one of the most frequent sources of human infection in developed countries. Here we evaluated the immunogenicity and protective efficacy of Salmonella Typhimurium $\chi 9718$ producing the Campylobacter jejuni $\mathrm{CjaA}$ protein as a chicken anti-Campylobacter vaccine. In this study chickens were orally immunized with a new generation $S$. Typhimurium strain $\chi 9718$ with regulated delayed attenuation in vivo and displaying delayed antigen expression. The immunization with the $S$. Typhimurium $\chi 9718$ strain producing $C$. jejuni $\mathrm{CjaA}$ antigen induced strong immune responses against $\mathrm{CjaA}$ in both serum $\mathrm{IgY}$ and intestinal IgA, however, it did not result in the significant reduction of intestinal colonization by Campylobacter strain. The low level of protection might arise due to a lack of T cell response. Our results demonstrated that a Salmonella strain with regulated delayed attenuation and displaying regulated delayed antigen expression might be an efficient vector to induce immune response against Campylobacter. It seems
\end{abstract}

Electronic supplementary material The online version of this article (doi:10.1007/s11274-013-1447-5) contains supplementary material, which is available to authorized users.

P. Łaniewski · A. Wyszyńska · E. K. Jagusztyn-Krynicka ( $\square)$ Department of Bacterial Genetics, Faculty of Biology, Institute of Microbiology, University of Warsaw, Miecznikowa 1, 02-096 Warsaw, Poland

e-mail: kjkryn@biol.uw.edu.pl

M. Kuczkowski · K. Chrząstek · A. Woźniak · A. Wieliczko Department of Epizootiology and Clinic for Birds and Exotic Animals, Faculty of Veterinary Medicine, Wroclaw University of Environmental and Life Sciences, pl. Grunwaldzki 45, 50-366 Wroclaw, Poland that an efficient anti-Campylobacter subunit vaccine should be multicomponent. Since $S$. Typhimurium $\chi 9718$ contains two compatible balanced-lethal plasmids, it can provide the opportunity of cloning several Campylobacter genes encoding immunodominant proteins. It may also be used as a delivery vector of eukaryotic genes encoding immunostimulatory molecules to enhance or modulate functioning of chicken immune system.

Keywords Campylobacter - CjaA · Chicken · Regulated delayed attenuation $\cdot$ Salmonella-delivered vaccine

\section{Introduction}

Food poisoning and diarrheal diseases in Europe and in the US continue to be a serious healthcare problem (Newell et al. 2010). Campylobacter spp. are generally regarded as the most common bacterial cause of gastroenteritis worldwide. The number of reported culture-confirmed human campylobacteriosis cases in the European Union (EU) was 212,064 in 2010. The rate of campylobacteriosis differs markedly among the EU countries, ranging from 0.04 to 200.58 per 100,000 individuals. Differences between countries cannot be directly compared and should be viewed with caution, since surveillance and reporting systems differ considerably from country to country (Janssen et al. 2008; EFSA 2012). Of particular concern are two species, Campylobacter jejuni and Campylobacter coli, which in 2010 accounted for the majority of intestinal human infections, 35.7 and $2.3 \%$ of reported cases, respectively. Other species reported included Campylobacter lari $(0.22 \%)$ and Campylobacter upsaliensis $(0.006 \%)$. The remaining Campylobacter cases were not characterized at the species level (EFSA 2012). 
Campylobacteriosis usually occurs sporadically from the consumption of contaminated food, mainly poultry meat. Even though most cases of campylobacteriosis are sporadic, infrequent outbreaks are also reported and associated with drinking contaminated unpasteurized milk or water. Of these sources, broiler meat contaminated with Campylobacter is unquestionably the main source of human infections due to high levels of consumption (Silva et al. 2011; Newell and Fearnley 2003). In 2010, as in previous years, the proportions of Campylobacter-positive broiler meat samples varied widely among the EU countries (from 3.1 to $90 \%$ ), and the occurrence of bacteria remained at a high level throughout the food chain: from live animals to the meat retail level (EFSA 2012). This fact, in combination with the relatively low human infection dose, can explain why the majority of human campylobacteriosis cases are associated with handling of uncooked and/or consumption of undercooked poultry meat. According to the European Food Safety Authority (EFSA) Biological Hazards Panel, 50 to $80 \%$ cases of human campylobacteriosis may be attributed to the chicken reservoir (EFSA 2010). The prevention of human infections requires control measures at all stages of the food chain, from agricultural production on the farm, to processing, manufacturing and preparation of foods in both commercial establishment and the domestic environment. It was calculated that reducing the numbers of Campylobacter in the bird's intestines at slaughter by three $\log 10$ units, would reduce the public health risk by at least $90 \%$ (EFSA 2011). The efficacy of Campylobacter control in broilers using potentially available pre- and post-harvest intervention methods seems to be limited or difficult to sustain. Thus, assuming that biosecurity can never be fully effective, implementation of immunoprophylactic methods for chickens may be the most efficient strategy to decrease the number of human Campylobacter infections. Vaccination should reduce colonization of birds with Campylobacter and as a result, decrease the amount of bacteria entering the food chain.

Live bacterial vaccine vectors such as attenuated human intestinal bacteria like Salmonella, Shigella or Listeria are being extensively studied for mucosal immunization in the prevention of different infectious diseases. These microorganisms, when delivered through the oral route, can cross the lumen of the gut and then be taken up by macrophages and dendritic cells at local sites, which results in the stimulation of humoral as well as cell-mediated and mucosal immune responses (Schoen et al. 2004, 2008; Loessner et al. 2008). Live oral vaccines based on diverse serovars of attenuated Salmonella enterica strains recently have been the most frequently used vehicles for the delivery of heterologous antigens/antigenic epitopes or as a DNA delivery system (Hegazy and Hensel 2012; Panthel et al. 2008; Curtiss et al. 2007). Until now, Campylobacter antigens/epitopes were delivered into the chicken immune system by Salmonella strains which carried defined mutations affecting metabolism/regulatory functions or virulence factors. Five types of avirulent $S$. enterica sv. Typhimurium ( $S$. Typhimurium) strains ( $\triangle a r o A, \Delta f i M$, $\Delta$ spaS, $\Delta s s a U$ and $\Delta c y a \Delta c r p)$ and also an aroA, htrA attenuated $S$. enterica sv. Enteritidis strains were tested in chicken immunoprophylaxis as vectors for Campylobacter antigens (Buckley et al. 2010; Layton et al. 2011; Wyszynska et al. 2004).

Diverse Campylobacter antigens or epitopes of selected antigens delivered orally by attenuated Salmonella strains have been tested as candidates for subunit chicken vaccine: $\mathrm{Cj} 0982 \mathrm{c}(\mathrm{CjaA})$ - the solute-binding protein, components of the ABC transport system (Muller et al. 2005); Cj0113 $(\mathrm{CjaD})$ - the peptidoglycan-associated protein (Pal) anchored in the outer membrane, a component of the PalTol system responsible for maintaining cell wall integrity (Godlewska et al. 2009); Cj0921c (Peb1) - the aspartate/ glutamate binding protein, a component of the $\mathrm{ABC}$ transport system (Leon-Kempis Mdel et al. 2006); Cj0420 (ACE393) - a protein of unknown function, identified by proteomics (Prokhorova et al. 2006; Schrotz-King et al. 2007); Cj0817 (GlnH) - the putative glutamine binding ABC transporter (Parkhill et al. 2000); Cj1614 (ChuA)the outer membrane protein involved in iron uptake (Miller et al. 2009); and Cj1534 (bactoferritin) (Med-Vet-Net 2009)—for review see (Jagusztyn-Krynicka et al. 2009; de Zoete et al. 2007).

So far, CjaA protein has been the most frequently tested Campylobacter antigen. $\mathrm{CjaA}$ is an extracytoplasmic protein present in the proteomes of both clinical and environmental isolates. Crystallographic analyses of the Escherichia coli-produced $\mathrm{rCjaA}$ determined that $\mathrm{CjaA}$ binds a cysteine ligand (Muller et al. 2005). Additionally, the expression levels of the cjaA gene increase when bacterial cultures are grown on iron-deficient and solid media. Both of these facts suggest participation of $\mathrm{CjaA}$ in the in vivo colonization process. Additionally, $\mathrm{CjaA}$ protein is more abundant in the proteome of clinical Campylobacter isolates as compared to the proteome of the laboratory strain and is recognized by chicken maternal antibodies (Cordwell et al. 2008; Shoaf-Sweeney et al. 2008). All these characteristics make $\mathrm{CjaA}$ a favorable candidate protein for subunit vaccine construction.

Here we evaluated the effectiveness of $\mathrm{CjaA}$ protein delivered by a new generation $S$. Typhimurium strain (double balanced-lethal host-vector system) with regulated 
delayed attenuation and displaying regulated delayed antigen expression for chicken immunization against $C$. jejuni. In this strain chromosomal deletions of house-keeping genes encoding proteins required for peptidoglycan synthesis i.e. aspartate-semialdehyde dehydrogenase (asd) and alanine racemases $(d a d B$ and $a l r)$ are complemented by wild-type copies of genes present on $\mathrm{Asd}^{+}$and $\mathrm{DadB}^{+}$plasmids, which eliminates drug-resistance markers in live vaccines (Wang et al. 2013; Xin et al. 2012). Additionally, the important features of these strains are regulated delayed attenuation in vivo and delayed antigen expression system. It displays wild-type phenotype when grown in vitro and at the time of oral vaccination (Curtiss et al. 2009; Li et al. 2009). Thus their ability to colonize host lymphoid tissue is similar to that of virulent strain. However, they become fully attenuated after host tissue colonization, where there is no free arabinose. The strategy to achieve regulated delayed attenuation relies on the use of the arabinose-regulated araC $\mathrm{P}_{\mathrm{BAD}}$ activator promoter. The original promoters of several genes which products are involved in metabolism or virulence were replaced with the tightly arabinose-regulated araC $\mathrm{P}_{\mathrm{BAD}}$ activator promoter. Regulated delayed in vivo synthesis of protective heterologous antigens has been achieved by using a chromosomal lactose repressor gene (lacl) under the transcriptional control of the arabinose-regulated $\operatorname{araC} \mathrm{P}_{\mathrm{BAD}}$ promoter (Wang et al. 2010). LacI negatively regulates the expression from trc promoter that drives the synthesis of heterologous antigens. In animal tissues, where arabinose is unavailable, the concentration of LacI decreases, thus allowing increased antigen synthesis.

\section{Materials and methods}

Bacterial strains, plasmids, media and growth conditions

Bacterial strains and plasmids used in this study are listed in Table 1. E. coli strain TG1 was used as a host for preparation of recombinant plasmids possessing an antibiotic selection marker. E. coli strain $\chi 6212$, kindly given by Roy Curtiss III (Arizona State University, AZ, USA), was used as a host for $\mathrm{Asd}^{+}$balanced-lethal plasmids. E. coli BL21(DE3) was employed to overproduce recombinant proteins. S. Typhimurium strain $\chi 9718$, also provided by Roy Curtiss III, was used in animal experiments. All E. coli and $S$. Typhimurium strains were routinely cultured at $37{ }^{\circ} \mathrm{C}$ in Luria-Bertani (LB) broth (Sigma-Aldrich, St. Louis, MO) or on LB agar. Nutrient broth (NB, BD Difco, Franklin Lakes, NJ), which is devoid of sugars, was used for analysis of the regulated delayed antigen synthesis system and determination of lipopolysaccharide (LPS) profiles. Strains were grown in NB without sugars overnight and subcultured (1:100) into fresh NB without or with $0.2 \%$ L-(+)-arabinose (Sigma-Aldrich) or $0.2 \%$ D-(+)-mannose (Sigma-Aldrich) for a second passage. LPS was prepared and visualized by silver staining in $12 \%$ polyacrylamide gels as described previously (Hitchcock and Brown 1983). MacConkey agar plates with $1 \%$ maltose (without or with $0.2 \%$ arabinose) or $1 \%$ arabinose were used to indicate fermentation of appropriate sugars. LB agar plates supplemented with $20 \mu \mathrm{M}$ deferoxamine mesylate (iron chelator) (without or with $0.2 \%$ arabinose) were used to test the growth in iron-depleted environment. Motility was observed in soft agar plates (LB solidified with $0.4 \%$ agar). Diaminopimelic acid (DAP, $50 \mu \mathrm{g} / \mathrm{ml}$, Sigma-Aldrich) was added for growth of $\mathrm{Asd}^{-}$ strains. For animal experiments, plasmid-carrying $S$. Typhimurium $\chi 9718$ strain was cultured in LB broth supplemented with $0.2 \% \mathrm{D}-(+)$-mannose and $0.2 \% \mathrm{~L}-(+)-$ arabinose to display appropriate phenotype at time of inoculation (see Discussion). An overnight culture was diluted 1:50 and grown to an optical density of $\sim 0.6$, which was measured using Biophotometer (Eppendorf, Hamburg, Germany), then bacteria were harvested by centrifugation at $4{ }^{\circ} \mathrm{C}(5,000 \times g$ for $15 \mathrm{~min})$ and the pellet was resuspended in phosphate-buffered saline (PBS).

Campylobacter jejuni strain 81-176 was the source of the $c j a A$ (cj0982c) gene. C. jejuni strain Wr1, isolated from a chicken, was employed in animal experiments. $C$. jejuni strains were routinely grown on Blood Agar Base No. 2 (Merck, Darmstadt, Germany) plates supplemented with $5 \%$ horse blood and "Campylobacter Selective Supplement (Blaser-Wang)" (Oxoid, Basingstoke, UK) at $37{ }^{\circ} \mathrm{C}$ or $42{ }^{\circ} \mathrm{C}$ for $16-24 \mathrm{~h}$ under microaerobic conditions $(5 \%$ $\mathrm{O}_{2}, 10 \% \mathrm{CO}_{2}, 85 \% \mathrm{~N}_{2}$ ). Campylobacter charcoal differential agar (CCDA) supplemented with "Modified Preston Campylobacter Selective Supplement" (Oxoid) was used to enumerate $C$. jejuni recovered from chickens.

\section{General DNA procedures}

DNA manipulations-i.e. plasmid and genomic DNA isolation, restriction enzyme digestions, ligations and other DNA-modifying reactions-were carried out as described by Sambrook and Russel (2001) or were performed according to the manufacturers' instructions (A \& A Biotechnology, Gdynia, Poland; Fermenters, Vilnius, Lithuania). Synthesis of primers (Table 2) and DNA sequencing were performed by Genomed S.A. (Warsaw, Poland). Polymerase chain reactions (PCR) were carried out under standard conditions with HotStar HiFidelity Polymerase (Qiagen, Hilden, Germany), possessing proofreading activity. Recombinant plasmids were introduced into $E$. coli and $S$. Typhimurium cells by transformation or electroporation, respectively. 
Table 1 Bacterial strains and plasmids used in this study

\begin{tabular}{|c|c|c|}
\hline Name & Relevant characteristics & Source or reference \\
\hline \multicolumn{3}{|l|}{ E. coli strains } \\
\hline TG1 & F' (traD36 proAB ${ }^{+}$lacl $^{\mathrm{q}}$ lacZ $\left.\Delta \mathrm{M} 15\right)$ supE hsd $\Delta 5$ thi $\Delta($ lac-proAB) & (Sambrook and Russel 2001) \\
\hline BL21(DE3) & $\mathrm{F}^{-}$ompT hsdSB $\left(\mathrm{r}_{\mathrm{B}}^{-} \mathrm{m}_{\mathrm{B}}^{-}\right)$gal dcm (DE3) & Novagen \\
\hline$\chi 6212$ & $\begin{array}{c}\mathrm{F}^{-} \lambda^{-} \varphi 80 \Delta(\text { lacZYA-argF }) \text { endA1 recAl hsdR17 } \\
\text { deoR thi-1 glnV44 gyrA96 relA1 } \triangle \text { asdA4 }\end{array}$ & (Nakayama et al. 1988) \\
\hline \multicolumn{3}{|c|}{ S. Typhimurium strains } \\
\hline$\chi 9718$ & $\begin{array}{l}\Delta p m i-2426 \Delta(\text { gmd-fcl })-26 \Delta_{\text {fur81 }}:: \mathrm{TT} \text { araC } \\
\mathrm{P}_{\mathrm{BAD}} \text { fur } \Delta \mathrm{P}_{\text {crp527 }}:: \text { TT araC } \mathrm{P}_{\mathrm{BAD}} \text { crp } \Delta a s d A 27:: \mathrm{TT} \\
\text { araC } \mathrm{P}_{\mathrm{BAD}} \text { c2 } 2 \text { araE25 } \Delta \text { araBAD23 } \Delta \text { relA198::araC } \\
\mathrm{P}_{\mathrm{BAD}} \text { lacI TT } \Delta \text { sopB1825 } \Delta a g f B A C 811 \Delta \text { alr } 3 \\
\Delta \text { dadB4 } \Delta \text { fliC180 }\end{array}$ & R. Curtiss III \\
\hline \multicolumn{3}{|l|}{ C. jejuni strains } \\
\hline $81-176$ & $\begin{array}{l}\text { Wild type; isolated from a child with bloody diarrhea } \\
\text { during an outbreak in Minnesota (USA); pVir, pTet } \\
\left(\mathrm{Tc}^{\mathrm{R}}\right) \text {; Lior 5; Penner } 23 / 26\end{array}$ & (Korlath et al. 1985) \\
\hline Wr1 & Wild type; isolated from a chicken; good colonizer & This study \\
\hline \multicolumn{3}{|l|}{ Plasmids } \\
\hline pGEM-T Easy & $A p^{R} ; T$ vector for cloning PCR products & Promega \\
\hline pBluescript II KS & $\mathrm{Ap}^{\mathrm{R}}$; general cloning vector & Stratagene \\
\hline pYA3342 & $\mathrm{Asd}^{+} ; \operatorname{trc}$ promoter; expression vector & (Kang et al. 2002) \\
\hline $\mathrm{pET} 22 \mathrm{~b}$ & $\mathrm{Ap}^{\mathrm{R}}$; lacI; overexpression vector & Novagen \\
\hline pUWM1050 & $c j a A$ in pGEM-T Easy & This study \\
\hline pUWM1161 & $c j a A$ in pYA3342 & This study \\
\hline pUWM1144 & $c j a A$ fragment (encoding protein without SS) in pBluescript II SK & This study \\
\hline pUWM1146 & pelB $_{S S^{-}}$cjaA-6xhis fusion in $\mathrm{pET} 22 \mathrm{~b}$ & This study \\
\hline
\end{tabular}

SS denotes signal sequence

Table 2 Primers used in this study

\begin{tabular}{llll}
\hline Name & Sequence $\left(5^{\prime} \rightarrow 3^{\prime}\right)$ & Orientation & Restriction site \\
\hline $1001 \mathrm{Nco}$ & CGTGCCATGGCAAAAATACTTCTAAGTG & Forward & NcoI \\
$1001 \mathrm{H}$ & CGTAAGCTTCAACTAAAGGGCAAAAAGC & Reverse & HindIII \\
$1001 \mathrm{BE}$ & ACGGATCCGGAATTCGGAGGAAATTCTGACTC & Forward & BamHI/EcoRI \\
$1001 \mathrm{Xho}$ & ACCTCGAGAATTTTTCCACCTTCAATCAC & Reverse & XhoI \\
\hline
\end{tabular}

Nucleotides underlined denote restriction enzyme sites used for cloning. Nucleotides bolded are complementary to the $C$. jejuni 81-176 chromosome

Construction of the $\mathrm{CjaA}^{+}$recombinant plasmid

The cjaA gene was amplified from $C$. jejuni $81-176$ chromosomal DNA with primers $1001 \mathrm{Nco}$ and $1001 \mathrm{H}$ (Table 2) and cloned into pGEM-T Easy (Promega, Madison, WI). Thereafter, the resulting plasmid, pUWM1050, was digested with NcoI and HindIII restriction enzymes and a $0.9 \mathrm{~kb}$ DNA fragment was inserted into pYA3342. The resulting plasmid pUWM1161 was verified by sequencing and transformed into E. coli strain $\chi 6212$. Protein production was confirmed by a Western blot using previously obtained rabbit polyclonal anti-rCjaA serum (Pawelec et al. 2000).
Preparation of subcellular fractions

Periplasmic proteins were released from the cells using an osmotic-shock procedure (Ausubel et al. 1989). After decanting the periplasmic fraction, bacterial pellets were resuspended in PBS and sonicated to release the cell contents. Subsequently cell wall debris was removed by centrifugation $\left(4,000 \times g, 4{ }^{\circ} \mathrm{C}, 20 \mathrm{~min}\right)$ and the supernatants were ultracentrifuged $\left(100,000 \times g, 4{ }^{\circ} \mathrm{C}, 1 \mathrm{~h}\right)$ to separate membrane and cytoplasmic fractions. Finally, the cell envelope was fractionated into inner and outer membranes by selective solubilization of the inner 
membrane with $N$-lauroylsarcosine sodium salt (MP Biomedicals, Solon, OH) (Filip et al. 1973).

\section{SDS-PAGE and Western blotting}

SDS-PAGE and Western blotting procedures were done by standard techniques. Blots were developed with nitro blue tetrazolium chloride/5-bromo-4-chloro-3'-indolyl phosphate (Sigma-Aldrich) as a substrate, using previously obtained rabbit polyclonal anti-rCjaA serum (Pawelec et al. 2000) or rabbit anti-GroEL antibodies (Sigma-Aldrich) as primary antibodies and mouse anti-rabbit $\operatorname{IgG}$ alkaline phosphatase conjugate (Sigma-Aldrich) as secondary antibodies. Density of protein bands on the Western blots was calculated using ImageJ software (National Institutes of Health, Bethesda, MD).

\section{Animal supply and housing}

Straight run Cobb 500 broiler chickens were obtained on the day of hatch from a local hatchery. Birds were housed in an animal facility in separate cages for each group and given water and feed ad libitum. Chickens were confirmed to be culture-negative for Campylobacter by cloacal swabbing. All animal experiments were carried out according to the ethical standards with the approval (No. 915/2008) of the First Warsaw Local Ethics Committee for Animal Experimentation (Warsaw, Poland).

\section{Immunization and challenge regimen}

1-day-old chickens were inoculated orally into the crop with $1 \mathrm{ml}$ of PBS containing $\sim 10^{8} \mathrm{CFU}$ of $S$. Typhimurium strain $\chi 9718$ harboring two recombinant plasmids: pUWM1161 (Asd $^{+}$vector carrying the cjaA gene) and pYA4346 ("empty" DadB ${ }^{+}$vector) and boosted with the same strain and dose 2 weeks later. A group of birds inoculated with PBS was used as a control. At 4 weeks of age birds (a half from each group) were orally challenged with $\sim 10^{5} \mathrm{CFU}$ of $C$. jejuni wild-type strain Wr1. At week 1 and 2 post challenge, 6 birds (from each group) were euthanized and samples of cecum were collected. Dilutions of the content were made in PBS and plated onto CCDA plates for enumeration of $C$. jejuni. To monitor humoral immune response, 8 birds (from each group) were sacrificed at weekly intervals for up to 6 weeks post immunization and samples of serum and gut secretion were collected at the post mortem examination. On day 1 post hatch the same number of unvaccinated birds were also euthanized. Samples were collected from $C$. jejuni-challenged as well as non-infected birds. Blood samples were taken after decapitation. Following centrifugation, sera were collected and stored at $-20^{\circ} \mathrm{C}$. To isolate secretion
IgA the mucus from the distal part of the ileum (a segment of approximately $5 \mathrm{~cm}$ ) was collected. The mucus samples were diluted with PBS containing $0.05 \%$ Tween 20 and soybean trypsin inhibitor $(0.1 \mathrm{mg} / \mathrm{ml})$ (dilution $1: 5)$. Samples were shaken for $30 \mathrm{~min}$ at $4{ }^{\circ} \mathrm{C}$, centrifuged at $20,000 \times g$ for $15 \mathrm{~min}$ at $4{ }^{\circ} \mathrm{C}$ and afterwards the supernatant was collected and stored at $-20{ }^{\circ} \mathrm{C}$. To determine population of lymphocytes, 8 birds (from immunized and control groups) were euthanized at 22, 31 and 35 days of age (i.e. 7, 16 and 20 days post booster) and spleens and cecal tonsils were collected and subjected to flow cytometric analysis.

\section{Antigen preparation}

A DNA fragment, encoding the mature CjaA protein, i.e. without the signal sequence, was amplified from $C$. jejuni 81-176 chromosomal DNA with primers 1001BE and 1001Xh (Table 2) and cloned into pBluescript II SK. The resulting plasmid, pUWM1144, was transformed into E. coli strain TG1. Afterwards a $0.9 \mathrm{~kb}$ DNA fragment from pUWM1144 containing the cjaA gene was recloned into pET22b using BamHI and XhoI restriction enzymes. The pET22b vector carries a fragment of Erwinia carotovora pectate lyase $\mathrm{B}$ gene (pelB) to localize potential fusion proteins in periplasmic space. The resulting plasmid pUWM1146, which carries a fusion of $c j a A$ with a pelB fragment encoding the signal sequence at $3^{\prime}$-terminus and a DNA fragment encoding a $6 x \mathrm{His}$ tag at the $5^{\prime}$-terminus, was verified by sequencing and transformed into $E$. coli strain BL21(DE3) (Table 1). The 6xHis-tagged CjaA protein was overproduced by auto induction (Studier 2005). Induced bacteria were lysed by sonication and the recombinant protein was purified by immobilized metal affinity chromatography using Ni-NTA agarose (Sigma-Aldrich) following the manufacturer's protocol. Overexpression and all purification steps were monitored by SDS-PAGE. Thereafter, the protein was dialyzed extensively against $50 \mathrm{mM}$ carbonate/bicarbonate buffer $(\mathrm{pH}$ 9.6) and used in ELISA assays as a coating antigen.

\section{Enzyme-linked immunosorbent assay (ELISA)}

The level of the antibodies against $C$. jejuni $\mathrm{CjaA}$ protein in chicken intestinal secretions and sera was quantified by ELISA. Briefly, 96-well Maxisorp plates (Nunc, Rochester, $\mathrm{NY}$ ) were coated overnight at $4{ }^{\circ} \mathrm{C}$ with the purified recombinant $\mathrm{CjaA}$ protein $(5 \mu \mathrm{g}$ per well), washed three times with PBS containing $0.02 \%$ Tween 20 (SigmaAldrich), blocked for $1 \mathrm{~h}$ at $37{ }^{\circ} \mathrm{C}$ with PBS containing $2 \%$ bovine serum albumin (Sigma-Aldrich), washed as previously described and incubated for $1.5 \mathrm{~h}$ at room temperature with the diluted sera $(1: 800)$ or intestinal 
secretion samples (1:10). The plates were developed with 3,3',5,5'-tetramethyl benzidine (Sigma-Aldrich) using antichicken IgA horseradish peroxidase conjugate (Thermo Scientific, Rockford, IL) or rabbit anti-chicken IgY (whole molecule)-peroxidase (Sigma-Aldrich) (dilution 1:2000), respectively. The plates were incubated with the substrate for $25 \mathrm{~min}$ at room temperature and then the colorimetric reaction was stopped by adding $2 \mathrm{M} \mathrm{H}_{2} \mathrm{SO}_{4}$ (SigmaAldrich). Absorbance was measured at $450 \mathrm{~nm}$ using an ELISA reader (Biotek, Winooski, VT). Each sample was analyzed in duplicate.

Flow cytometry

Freshly collected cecal tonsil and spleen samples from all birds were immediately homogenized. Afterwards cells were separated in density gradients on Ficoll (Sigma Aldrich) at $720 \times g$ for $30 \mathrm{~min}$ at $4{ }^{\circ} \mathrm{C}$. Buffy coats were collected, placed in separate tubes, then washed in PBS and centrifuged twice at $720 \times g$ for $7 \mathrm{~min}$ at $4{ }^{\circ} \mathrm{C}$. For immunophenotypic analyses, aliquots of spleen and cecal tonsil lymphocytes $\left(1 \times 10^{6}\right.$ cells per $\left.\mathrm{ml}\right)$ were incubated with different mouse anti-chicken monoclonal antibodies: R-PE- CD3 (clone CT3), FITC- CD4 (clone CT-4), R-PE-CD8 $\alpha$ (clone CT-8), FITC-Bu-1 (clone AV20), FITC-TCR $\gamma \delta$ (clone TCR 1) (SouthernBiotech, Birmingham, AL) for $30 \mathrm{~min}$, and then washed three times with PBS. All samples were analyzed by flow cytometry (Becton-Dickinson Immunocytometry Systems, San Jose, CA). For each sample, 20,000 gated cells were acquired and the percentage of cells in the population was determined using CellQuest software (Becton-Dickinson Immunocytometry Systems).

Statistical analysis

Statistical analyses of the ELISA and colonization results were performed using Statistica 9.0 (StatSoft, Krakow, Poland) or GraphPad Prism 6 (GraphPad Software, Inc., San Diego, CA). The significance of differences between the obtained values was appraised using one-way analysis of variance (ANOVA) followed by the post hoc Tukey test. $p$ values $<0.05$ were considered significant.

\section{Results}

Production of $\mathrm{CjaA}$ in S. Typhimurium with regulated delayed attenuation

Plasmid pUWM1161 was used to express $C$. jejuni cjaA gene in $S$. Typhimurium carrier cells. This recombinant plasmid, a derivative of the balanced-lethal $\mathrm{Asd}^{+}$vector pYA3342, harbors the cjaA gene under the control of trc promoter. This strong promoter is regulated by the lacI gene cloned into the chromosome of the host as a $\mathrm{P}_{\mathrm{BAD}}$ lacI TT cassette. Thus, the level of the CjaA synthesis is dependent on the presence of arabinose, which should minimize the negative effect of antigen overproduction on the host strain during the early phase of infection.

First pUWM1161 was introduced into E. coli $\chi 6212$ by transformation. Western blot experiments with specific rabbit anti-rCjaA antibodies showed a high level of protein production (data not shown). Next pUWM1161 was moved into $S$. Typhimurium strain $\chi 9718$ (pYA4346) carrying a regulated delayed expression cassette in the chromosome by electroporation. This strain also harbors an "empty" balanced-lethal vector, pYA4346, which can be used in future work to produce additional antigens in the same host. Western blot analysis with specific anti-rCjaA antibodies showed induction of CjaA synthesis, when cells were grown in medium without arabinose (Fig. 1). The experiment confirmed the functionality of the delayed antigen expression system.

In a separate experiment, the effect of $c j a A$ overexpression on regulated delayed attenuation of the host strain was also determined. The rationale was to check whether the high amount of the heterologous antigen disrupted the host strain phenotype. To do this we verified the selected phenotypic characteristics of $S$. Typhimurium strain $\chi 9718$ (pYA4346, pUWM1161): i.e. LPS profile, sugar fermentation, Fur synthesis and motility. We showed that the expression of the cjaA does not influence the regulated delayed attenuation of the $S$. Typhimurium host cells (Fig. S1).

Localization of CjaA in $S$. Typhimurium cells

CjaA localization in $S$. Typhimurium strain, a host of the $C$. jejuni cjaA gene, was analyzed by a standard cell

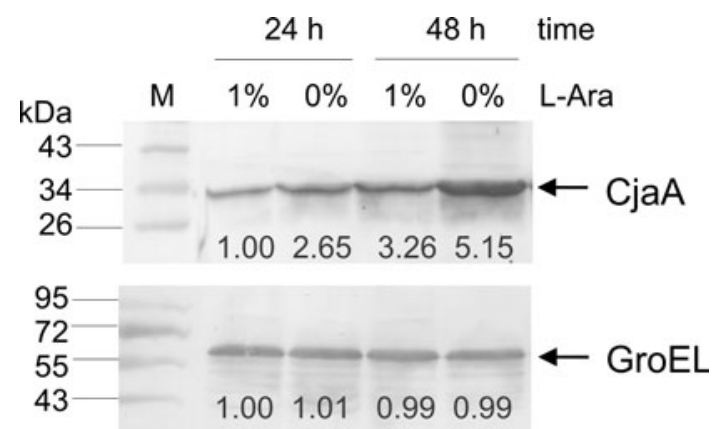

Fig. 1 CjaA synthesis in $S$. Typhimurium strain $\chi 9718$ (pYA4346, pUWM1161). Bacteria were grown in nutrient broth containing the indicated concentration of arabinose for 24-48 h. Cells were sonicated and equal amounts of protein $(c .10 \mu \mathrm{g})$ were electrophoresed on a $12 \%$ SDS-PAGE gel, transferred onto nitrocellulose and probed with anti-rCjaA antibodies. Lane $\mathrm{M}$ : molecular weight markers. The numbers denote the densities of each band, quantified using ImageJ software (National Institutes of Health, Bethesda, MD) 
fractionation procedure. Proteins derived from different cell compartments were analyzed by Western blot experiments using specific rabbit anti-rCjaA antibodies. CjaA protein was predominantly present in the inner membrane, as in a natural host, but it was also found in the cell periplasmic fraction. However, high amount of $\mathrm{CjaA}$ was also present in the cell cytoplasm probably due to overproduction of this protein (Fig. S2).

Antibody and cell-mediated immune response in vaccinated chickens

To analyze the antibody response of chicks to $\mathrm{CjaA}$ delivered by the attenuated $S$. Typhimurium strain two independent animal experiments were performed. In each experiment 1-day-old chicks were orally immunized with approximately $10^{8}$ CFU of $S$. Typhimurium $\chi 9718$ (pYA4346, pUWM1161) at day 1 and again with the same dose 2 weeks later. The control nonimmunized chicks were given sterile PBS. Half of the chicks from immunized group and from an age-matched control group were challenged with $10^{5}$ bacterial cells of a broiler-isolated $C$. jejuni strain 2 weeks after the booster. Serum IgY and mucosal IgA antibody responses against $\mathrm{CjaA}$ were measured by ELISA assays using Histagged $\mathrm{CjaA}(\mathrm{rCjaA})$ as a coating antigen.

Analysis of the serum samples obtained from 1-day-old chicks showed relatively high levels of specific IgY which decreased by week 2. This phenomenon confirms earlier reports that $\mathrm{CjaA}$ is recognized by maternal antibodies. Oral immunization with the recombinant $S$. Typhimurium vector resulted in induction of increasingly high IgY antibody responses to $\mathrm{CjaA}$ (Fig. 2). Significant differences between IgY levels present in the serum taken from control and immunized birds were observed at weeks 5 and 6 (i.e. 1 or 2 weeks after the booster). In both experiments the level of specific IgY antibodies peaked at week 6 (i.e. 4 weeks after the booster).

Analysis of the intestinal samples also showed that the immunization resulted in induction of specific mucosal IgA antibodies. The level of IgA consistently increased in both experiments starting at 1 week after the booster. The highest titers of mucosal IgAs in immunized chicks were observed likewise 4 weeks after the booster. It was also observed that Campylobacter challenge triggers chicken immune response. In both experiments, after the challenge, the level of IgYs in serum of nonimmunized birds was higher compared to non-infected birds. In one experiment, after the challenge an additional increase of IgY titers was also observed in infected birds, which had been previously immunized, compared to immunized birds that were not infected with $C$. jejuni.

To monitor induction of different type lymphocytes, samples of spleen and cecal tonsils were collected from the immunized and the control birds at days 22, 31 and 35 of the experiment (i.e. at days 7, 16 and 20 after the booster). The percentage of lymphocytes in each organ was determined by flow cytometry analysis. To identify populations of $\mathrm{T}$ cells (including all mature $\mathrm{T}$ lymphocytes as well as subpopulations of cytotoxic and helper/regulatory $\mathrm{T}$ cells) and $\mathrm{B}$ cells, monoclonal antibodies recognizing specific cell-surface antigens, respectively, CD3, CD4, CD8 $\alpha$ and $\mathrm{Bu}-1$ were used. Additionally, population of $\gamma \delta \mathrm{T}$ cells, which seem not to require major-histocompatibility-complex presentation, was determined with specific antiTCR $\gamma \delta$ antibodies. Results are summarized in Fig. 3. It was demonstrated that the percentage of B cells (expressing Bu-1 cell-surface antigen) in spleen and cecal tonsils of immunized birds was higher than that found in the control group at days 22. Similar results were obtained in cecal tonsils at days 31 . However, the percentage of $\mathrm{T}$ cells was not significantly different between the immunized and the control group.

\section{C. jejuni colonization in vaccinated chickens}

To analyze the ability of attenuated $S$. Typhimurium strain $\chi 9718$ (pYA4346, pUWM1161) producing CjaA antigen to reduce the colonization of birds by wild-type Campylobacter, immunized chicks were challenged with $10^{5}$ bacterial cells of a heterologous broiler-isolated $C$. jejuni strain 2 weeks after they were boosted. Challenge experiments were preceded by studies showing that chicks were not already colonized by Campylobacter. Cecal load was determined in euthanized birds as this method is more reliable than sampling of feces or cloacal swabs. The results of two independent experiments are shown in Fig. 4. Immunization with $S$. Typhimurium strain $\chi 9718$ (pYA4346, pUWM1161) hardly reduced the level of intestinal colonization by Campylobacter at two and 4 weeks after the challenge compared to the control group. However, the differences of the colonization levels are not statistically significant.

\section{Discussion}

Numbers of differently attenuated Salmonella strains have recently been generated and used as delivery vectors of recombinant heterologous antigens from bacterial, parasitic, viral, and tumor sources for human and animal immunization. In our laboratory we are interested in developing anti-Campylobacter vaccine for poultry. This is an area where using $S$. Typhimurium as a vector may be valuable since Salmonella sp. chicken infections constitute an important veterinary problem and such a vaccine may protect birds from both Salmonella and Campylobacter 

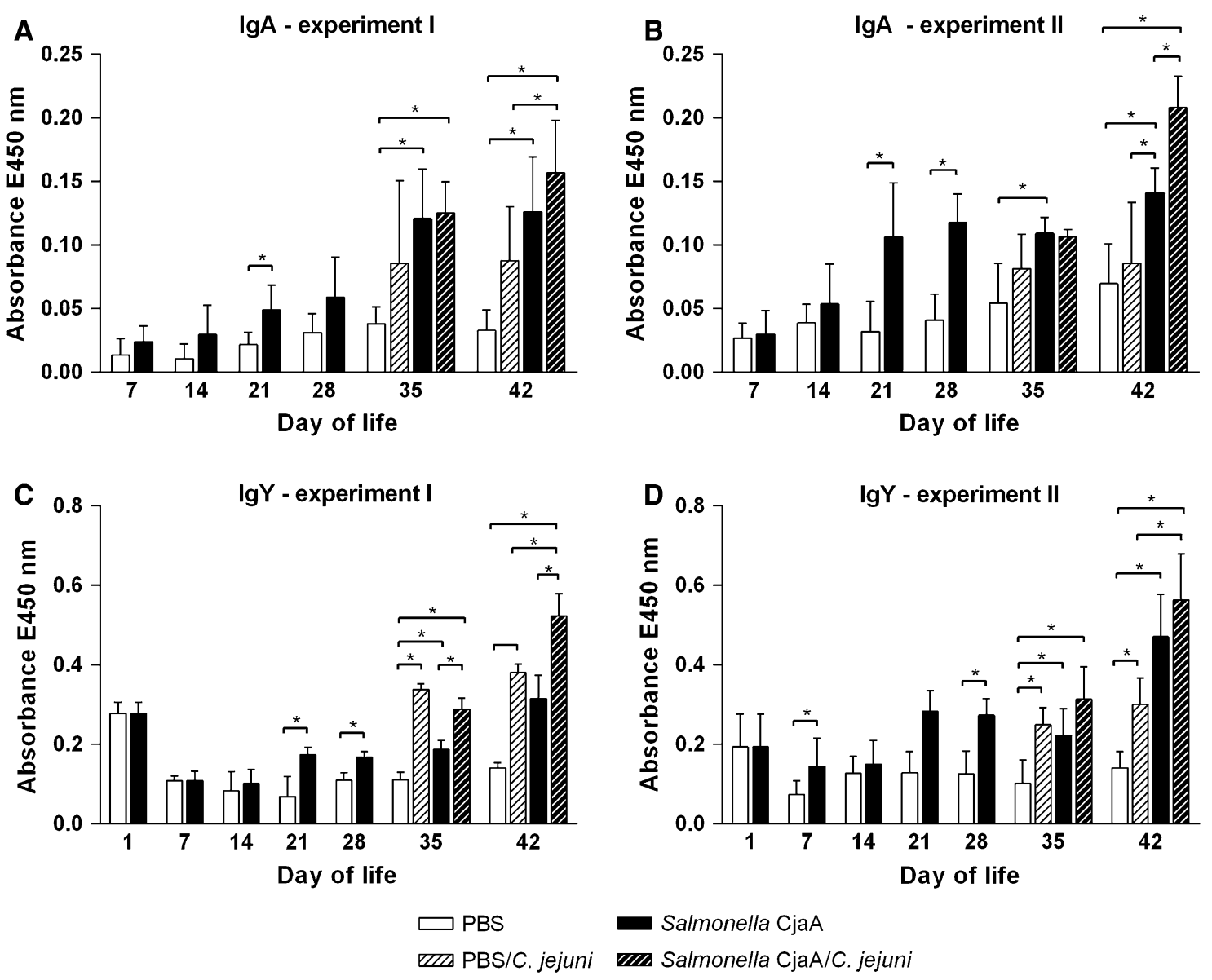

Fig. 2 Immune responses of chickens vaccinated with $S$. Typhimurium strains: $\chi 9718$ (pYA4346, pUWM1161). Levels of serum IgY and mucosal sIgA antibodies specifically recognizing CjaA antigen were determined by ELISA. Chickens were given two doses of vaccine strains at 1 and 14 days post hatch (solid bars). Some birds were also infected with $C$. jejuni at 28 days of life (hatched bars). Control birds were given PBS (open bars). Serum and intestinal

infections. So far, all S. enterica strains, tested on chickens as anti-Campylobacter vaccines, have been rendered avirulent by deleting chromosomal genes involved in metabolic pathways or genes coding regulatory proteins. It was documented that a mean of attenuation influences immunogenicity and efficacy of Salmonella vaccine strains. Thus, in this study we evaluated the immunogenicity and protective efficacy of $S$. Typhimurium $\chi 9718$ producing the highly immunogenic $C$. jejuni CjaA protein. This vector strain is a member of Salmonella vaccine strains named RASV RDAS (recombinant attenuated Salmonella vaccine; regulated delayed antigen synthesis). The two main features of these strains are regulated delayed attenuation in vivo and delayed antigen expression system. Strains, that belong to this family, display wild-type phenotype when grown in vitro and at the time of oral vaccination. Thus their ability to colonize host lymphoid tissue is similar to that of virulent strain. However, they become fully

samples were collected at the specified days of chicken life. Purified $\mathrm{rCjaA}$ protein was used as a coating antigen. Serum samples were diluted 1:800 and intestinal secretion samples 1:10. Absorbance values represent a mean of 8 birds \pm SD per time interval. A statistical analysis was carried out using the ANOVA Tukey's post hoc test. Asterisk indicates significant difference $(p<0.05)$ between groups

attenuated after host tissue colonization (Curtiss et al. 2009; Li et al. 2009). Some RASV RDAS strains have already been tested as a delivery vector of heterologous antigens (PspA of Streptococcus pneumonia and LcrV of Yersinia pestis) into the mouse immune system and it has been documented that they are immunogenic and safe (Torres-Escobar et al. 2010; Branger et al. 2009; Li et al. 2009; Shi et al. 2010). It was recently shown that $S$. Typhimurium RASV RDAS strain with dual $\left(\mathrm{Asd}^{+} \mathrm{DadB}^{+}\right)$ balanced-lethal host-vector system expressing two pneumococcal antigens protected mice against challenge with virulent $S$. pneumoniae (Xin et al. 2012). Our work was the first attempt to employ $S$. Typhimurium $\chi 9718$ harboring a C. jejuni antigen as a vector for chick immunization against Campylobacter colonization. The $C$. jejuni cjaA gene was cloned into plasmid pYA3342 carrying the asd gene, which complements a lethal asd deletion introduced into the host chromosome. This system enables cloning of foreign genes 

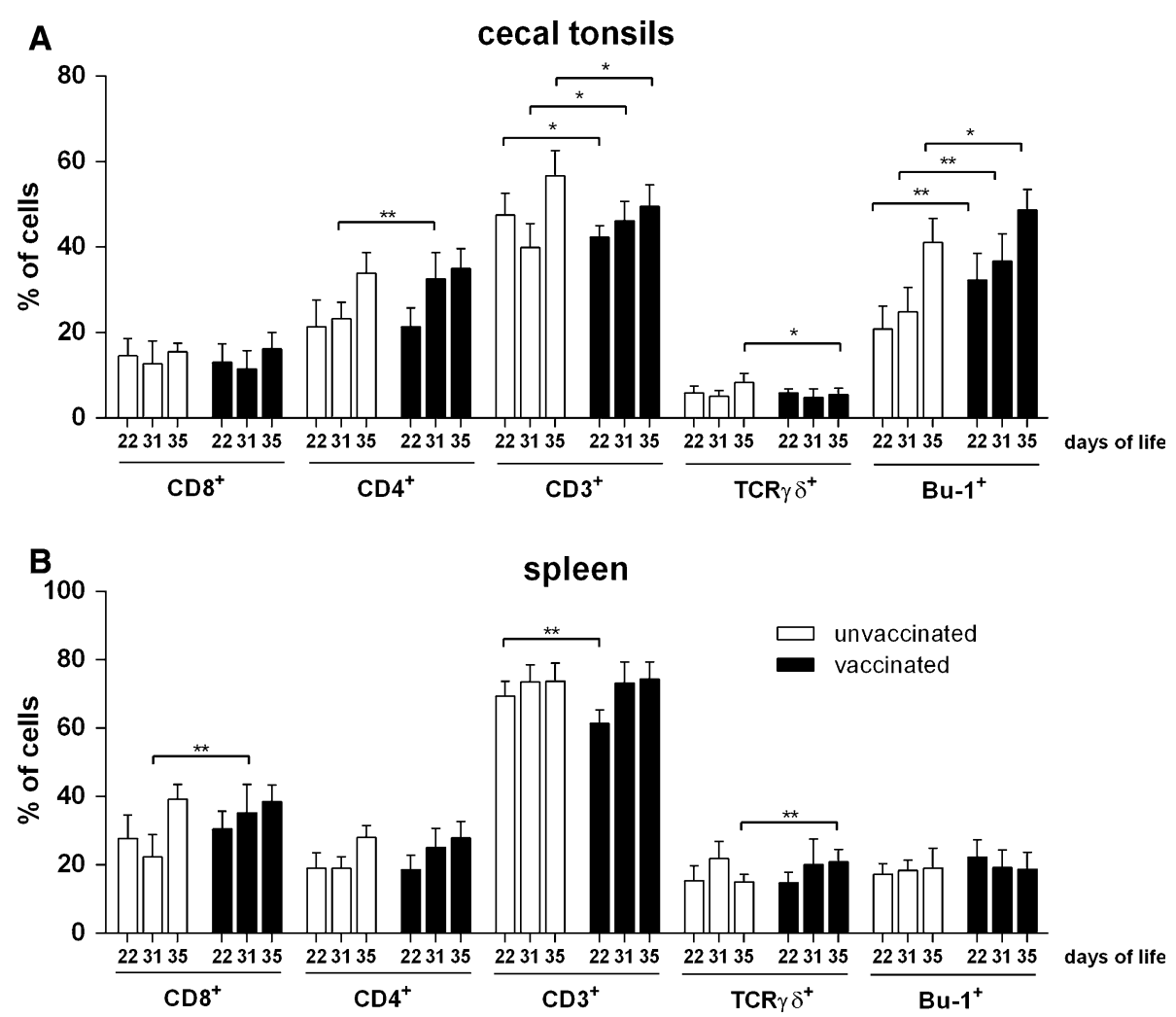

Fig. 3 The percentage of lymphocytes in spleen and cecal tonsils of chickens vaccinated with $S$. Typhimurium strain $\chi 9718$ (pYA4346, pUWM1161). Chickens were given two doses of vaccine strains at 1 and 14 days post hatch (solid bars). Control birds were given PBS (open bars). Spleen and cecal tonsils samples were collected at the specified days of chicken life. Types of lymphocytes in population of spleen and cecal tonsils cells were determined by flow cytometry

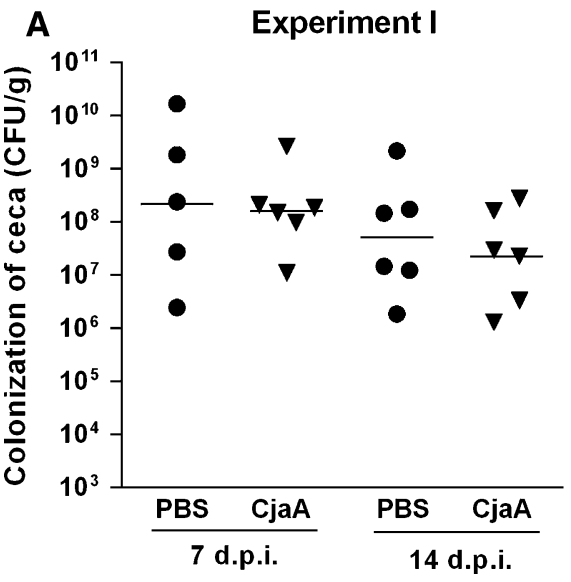

Fig. 4 Colonization of chickens vaccinated with $S$. Typhimurium strains: $\chi 9718$ (pYA4346, pUWM1161) after $C$. jejuni challenge. Chickens were given two doses of vaccine strains at 1 and 14 days after hatch and challenged with $C$. jejuni 2 weeks later. Control birds were given PBS. Viable $C$. jejuni cells were recovered from the ceca

without antibiotic selection. Additionally, the cjaA present on the recombinant plasmid pUWM1161 is under the control of trc promoter, which is regulated by LacI. At the using specific mouse anti-chicken monoclonal antibodies: R-PE- CD3 (clone CT-3), FITC- CD4 (clone CT-4), R-PE-CD8 $\alpha$ (clone CT-8), FITC-Bu-1 (clone AV20), FITC-TCR $\gamma \delta$ (clone TCR 1). Values represent a mean of 8 birds \pm SD per time interval. A statistical analysis was carried out using multiple unpaired $t$ test following Holm-Š́́dák test. Asterisks indicate significant difference between groups $(* * 0.001<p<0.01, * 0.01<p<0.05)$

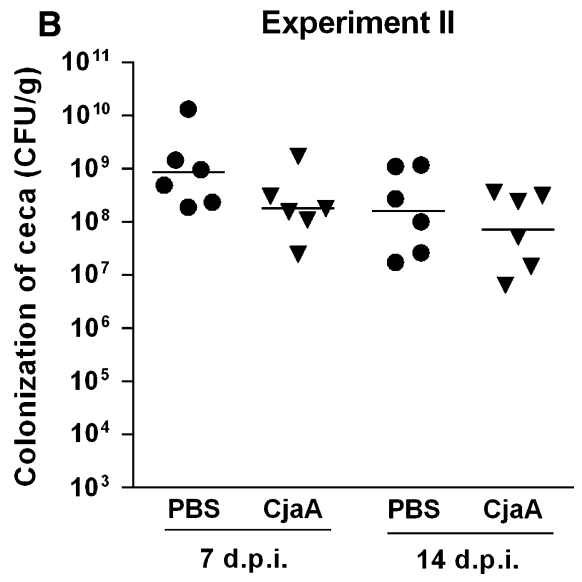

of chickens at specified days post infection (d.p.i.). Bacterial recoveries represent colonization levels of 6 birds per time interval. A geometric means for each group were denoted as bars. A statistical analysis was carried out using unpaired $t$ test. Differences between analyzed groups are not statistically significant

same time expression of the lacI gene is dependent on the presence of arabinose. Since arabinose is not available in animal tissue, the cjaA gene cloned in pUWM1161 is 
expressed at a high level when the Salmonella reaches the host immune system. We noticed a significant increase of the amount of $\mathrm{CjaA}$, when bacteria were grown without arabinose in vitro, but even in the presence of $1 \%$ of arabinose CjaA synthesis was not completely repressed (Fig. 1).

Since our experiment was the first attempt to employ S. Typhimurium $\chi 9718$ as a delivery vector for Campylobacter antigen we checked whether the production of foreign antigen disturbed the strain phenotype. As showed in supplementary materials, $S$. Typhimurium $\chi 9718$ producing $\mathrm{CjaA}$ retained the phenotype of regulated delayed attenuation.

Here, we observed that immunization with the $S$. Typhimurium RASV RDAS strain producing $\mathrm{CjaA}$ induced antibody response, both $\operatorname{IgY}$ in serum and intestinal $\operatorname{IgA}$, against the $C$. jejuni $\mathrm{CjaA}$ antigen. However, the induction of humoral and mucosal immune responses did not result in the significant reduction of $C$. jejuni colonization. Recently Buckley et al. have evaluated the effectiveness of chicken immunization against Campylobacter infection using a $S$. Typhimurium aroA mutant producing $\mathrm{CjaA}$ antigen fused to the $\mathrm{C}$-terminus of fragment $\mathrm{C}$ of tetanus toxin (Buckley et al. 2010). Oral immunization with this strain also resulted in only slight reduction (approximately 1.4 $\operatorname{logs}$ ) of intestinal colonization by $C$. jejuni after the challenge and this effect was not observed before week 4 post infection. The level of specific anti-CjaA IgYs in serum increased faster in the case of immunization with $S$. Typhimurium RASV RDAS (CjaA) in comparison with the effect observed after immunization with $S$. Typhimurium aroA. The kinetics of $\operatorname{IgA}$ induction cannot be compared between two experiments as we measured the level of intestinal specific IgAs and Buckley et al. evaluated the level of bile site IgAs. However, the increase of the specific intestinal $\operatorname{IgAs}$ (unchallenged birds) in our experiment was observed 1 week after the booster whereas Buckley et al. observed the highest level of specific $\operatorname{IgAs}$ in immunized but unchallenged birds 4 weeks after the booster. The rapid induction of the mucosal immune response is an important point since the effective vaccine delivered to immunologically immature birds should induce a sufficiently rapid response at the gut mucosal surface to protect the bird from challenge within 2-3 weeks of hatching.

Our previous work showed that using $S$. Typhimurium crp cya mutant expressing cjaA for bird immunization reduced the colonization by a heterologous $C$. jejuni strain by $6 \operatorname{logs}$ as compared to a nonimmunized control as early as 3 days after the challenge (Wyszynska et al. 2004). The observed inconsistencies with this study may be explained by differences in the host response to $C$. jejuni infection at a molecular level. In both experiments we used animals obtained from local hatchery. Recent comprehensive transcriptomic analysis of spleen and ceca RNA from two genetically distinct lines of broiler chickens revealed significant differences in response to $C$. jejuni infection. Interestingly the differences in gene expression were observed not only between two lines of chickens but also between birds within each line (Li et al. 2010, 2011, 2012).

Analysis of the lymphocyte population in spleen and cecal tonsils of chickens immunized with S. Typhimurium RASV RDAS confirmed stimulation of B cell production in both of lymphatic organs. However, CjaA delivered by attenuated Salmonella strain did not trigger T cell proliferation, which might have resulted in unsatisfactory protection of chickens from the Campylobacter challenge.

It is generally accepted that antigen localization may influence the protective efficacy when a Salmonella strain is used as a delivery vector. In the native host $\mathrm{CjaA}$ protein is found mainly in the inner membrane (Wyszynska et al. 2008). Here, the CjaA produced by attenuated $S$. Typhimurium strain was found as a periplasmic and innermembrane protein, whereas the CjaA fused to the C-terminus of fragment $\mathrm{C}$ of tetanus toxin produced by $S$. Typhimurium aroA was located in the cytoplasm (Buckley et al. 2010). Moreover, Layton et al. used CjaA delivered by Salmonella for chick immunization. In this case an $S$. Enteritidis aroA htrA strain produced a linear epitope of $\mathrm{CjaA}$ as a fusion to LamB, and additionally, the constructed chimeric protein was coexpressed with the immune-enhancing CD154 ligand, which plays a role in the regulation of the cellular immune response (Layton et al. 2011). Moderate reduction of colonization was observed in all three types of experiments. However, it is hard to draw conclusions from these various experiments, since so many other parameters, besides antigen localization, differed among the vaccination procedures.

Altogether, our results demonstrated that an attenuated Salmonella strain with regulated delayed attenuation and displaying a regulated delayed antigen expression might be an efficient vector to induce immune response against Campylobacter. However, all experiments so far clearly indicate that an efficient anti-Campylobacter subunit vaccine based on an attenuated Salmonella strain should be a multicomponent vaccine. $\mathrm{CjaA}$ is a promising component to include for the development of such a vaccine against Campylobacter, and S. Typhimurium $\chi 9718$, which contains two compatible plasmids, $\mathrm{Asd}^{+}$and $\mathrm{DadB}^{+}$, both constructed as a balanced-lethal vector-host systems, can provide the opportunity of cloning more Campylobacter genes. This host strain may also be used as a delivery vector of the immunostimulatory molecules to enhance or modulate functioning of chicken immune system.

Acknowledgments The work was supported by a grant from the Polish Ministry of Science and Higher Education (No N N302 
236838). We are grateful to Prof. R. Curtiss III for providing the Salmonella strains. We thank J. Hansen for his critical reading of the manuscript.

Open Access This article is distributed under the terms of the Creative Commons Attribution License which permits any use, distribution, and reproduction in any medium, provided the original author(s) and the source are credited.

\section{References}

Ausubel FM, Brent R, Kingston RE, Moore DD, Seidman JG, Smith JA, Struhl K (1989) Current protocols in molecular biology. Wiley \& Sons, New York

Branger CG, Torres-Escobar A, Sun W, Perry R, Fetherston J, Roland KL, Curtiss R 3rd (2009) Oral vaccination with LcrV from Yersinia pestis KIM delivered by live attenuated Salmonella enterica serovar Typhimurium elicits a protective immune response against challenge with Yersinia pseudotuberculosis and Yersinia enterocolitica. Vaccine 27(39):5363-5370. doi:10. 1016/j.vaccine.2009.06.078

Buckley AM, Wang J, Hudson DL, Grant AJ, Jones MA, Maskell DJ, Stevens MP (2010) Evaluation of live-attenuated Salmonella vaccines expressing Campylobacter antigens for control of $C$. jejuni in poultry. Vaccine 28(4):1094-1105. doi:10.1016/j. vaccine.2009.10.018

Cordwell SJ, Len AC, Touma RG, Scott NE, Falconer L, Jones D, Connolly A, Crossett B, Djordjevic SP (2008) Identification of membrane-associated proteins from Campylobacter jejuni strains using complementary proteomics technologies. Proteomics 8(1):122-139. doi:10.1002/pmic.200700561

Curtiss RI, Zhang X, Wanda SY, Kang HY, Konjufca V, Li Y, Gunn B, Wang S, Scarpellini G, Lee IS (2007) Induction of host immune responses using Salmonella-vectored vaccines. In: Brogden KA, Minion FC, Cornick N et al (eds) Virulence mechanisms of bacterial pathogens. ASM Press, Washington, DC, pp 297-313

Curtiss R 3rd, Wanda SY, Gunn BM, Zhang X, Tinge SA, Ananthnarayan V, Mo H, Wang S, Kong W (2009) Salmonella enterica serovar Typhimurium strains with regulated delayed attenuation in vivo. Infect Immun 77(3):1071-1082. doi:10. 1128/IAI.00693-08

de Zoete MR, van Putten JP, Wagenaar JA (2007) Vaccination of chickens against Campylobacter. Vaccine 25(30):5548-5557. doi:10.1016/j.vaccine.2006.12.002

EFSA (2010) EFSA panel on biological hazards (BIOHAZ); scientific opinion on quantification of the risk posed by broiler meat to human campylobacteriosis in the EU. 8(1):1437: [89 pp]. doi: 10.2903/j.efsa.2010.1437

EFSA (2011) EFSA panel on biological hazards (BIOHAZ). Scientific opinion on Campylobacter in broiler meat production: control options and performance objectives and/or targets at different stages of the food chain. 9(4):2105: [141 pp.]. doi:10. 2903/j.efsa.2011.2105. Available online: www.efsa.europa.eu/ efsajournal

EFSA (2012) The European Union summary report on trends and sources of zoonoses, zoonotic agents and food-borne outbreaks in the European Union in 2010. 10(3):2597:[442 pp.] doi:10. 2903/j.efsa.2012.2597. Available online: www.efsa.europa.eu/ efsajournal

Filip C, Fletcher G, Wulff JL, Earhart CF (1973) Solubilization of the cytoplasmic membrane of Escherichia coli by the ionic detergent sodium-lauryl sarcosinate. J Bacteriol 115(3):717-722
Godlewska R, Wisniewska K, Pietras Z, Jagusztyn-Krynicka EK (2009) Peptidoglycan-associated lipoprotein (Pal) of Gramnegative bacteria: function, structure, role in pathogenesis and potential application in immunoprophylaxis. FEMS Microbiol Lett 298(1):1-11. doi:10.1111/j.1574-6968.2009.01659.x

Hegazy WA, Hensel M (2012) Salmonella enterica as a vaccine carrier. Future Microbiol 7(1):111-127. doi:10.2217/fmb.11.144

Hitchcock PJ, Brown TM (1983) Morphological heterogeneity among Salmonella lipopolysaccharide chemotypes in silver-stained polyacrylamide gels. J Bacteriol 154(1):269-277

Jagusztyn-Krynicka EK, Laniewski P, Wyszynska A (2009) Update on Campylobacter jejuni vaccine development for preventing human campylobacteriosis. Expert Rev Vaccines 8(5):625-645. doi:10.1586/erv.09.21

Janssen R, Krogfelt KA, Cawthraw SA, van Pelt W, Wagenaar JA, Owen RJ (2008) Host-pathogen interactions in Campylobacter infections: the host perspective. Clin Microbiol Rev 21(3):505-518. doi:10.1128/CMR.00055-07

Kang HY, Srinivasan J, Curtiss R 3rd (2002) Immune responses to recombinant pneumococcal PspA antigen delivered by live attenuated Salmonella enterica serovar Typhimurium vaccine. Infect Immun 70(4):1739-1749

Korlath JA, Osterholm MT, Judy LA, Forfang JC, Robinson RA (1985) A point-source outbreak of campylobacteriosis associated with consumption of raw milk. J Infect Dis 152(3):592-596

Layton SL, Morgan MJ, Cole K, Kwon YM, Donoghue DJ, Hargis BM, Pumford NR (2011) Evaluation of Salmonella-vectored Campylobacter peptide epitopes for reduction of Campylobacter jejuni in broiler chickens. Clin Vaccine Immunol: CVI 18(3):449-454. doi:10.1128/CVI.00379-10

Leon-Kempis Mdel R, Guccione E, Mulholland F, Williamson MP, Kelly DJ (2006) The Campylobacter jejuni PEB1a adhesin is an aspartate/glutamate-binding protein of an $\mathrm{ABC}$ transporter essential for microaerobic growth on dicarboxylic amino acids. Mol Microbiol 60(5):1262-1275. doi:10.1111/j.1365-2958.2006. 05168.x

Li Y, Wang S, Scarpellini G, Gunn B, Xin W, Wanda SY, Roland KL, Curtiss R 3rd (2009) Evaluation of new generation Salmonella enterica serovar Typhimurium vaccines with regulated delayed attenuation to induce immune responses against PspA. Proc Natl Acad Sci USA 106(2):593-598. doi:10.1073/pnas.0811697106

Li X, Swaggerty CL, Kogut MH, Chiang HI, Wang Y, Genovese KJ, $\mathrm{He} \mathrm{H}$, Zhou H (2010) Gene expression profiling of the local cecal response of genetic chicken lines that differ in their susceptibility to Campylobacter jejuni colonization. PLoS One 5(7):e11827. doi:10.1371/journal.pone.0011827

Li XY, Swaggerty CL, Kogut MH, Chiang HI, Wang Y, Genovese KJ, He H, Pevzner IY, Zhou HJ (2011) Caecal transcriptome analysis of colonized and non-colonized chickens within two genetic lines that differ in caecal colonization by Campylobacter jejuni. Anim Genet 42(5):491-500. doi:10.1111/j.13652052.2010.02168.x

Li X, Swaggerty CL, Kogut MH, Chiang HI, Wang Y, Genovese KJ, He H, McCarthy FM, Burgess SC, Pevzner IY, Zhou H (2012) Systemic response to Campylobacter jejuni infection by profiling gene transcription in the spleens of two genetic lines of chickens. Immunogenetics 64(1):59-69. doi:10.1007/s00251-011-0557-1

Loessner H, Endmann A, Leschner S, Bauer H, Zelmer A, zur Lage S, Westphal K, Weiss S (2008) Improving live attenuated bacterial carriers for vaccination and therapy. Int $\mathrm{J}$ Med Microbiol 298(1-2):21-26. doi:10.1016/j.ijmm.2007.07.005

Med-Vet-Net (2009) Workshop on immunity to and vaccination against Campylobacter jejuni in chickens. In: Med-Vet-Net Workpackage 34, Veterinary Laboratories Agency, New Haw, Addlestone, Surrey KT15 3NB, UK 
Miller CE, Williams PH, Ketley JM (2009) Pumping iron: mechanisms for iron uptake by Campylobacter. Microbiology 155(Pt 10):3157-3165. doi:10.1099/mic.0.032425-0

Muller A, Thomas GH, Horler R, Brannigan JA, Blagova E, Levdikov VM, Fogg MJ, Wilson KS, Wilkinson AJ (2005) An ATPbinding cassette-type cysteine transporter in Campylobacter jejuni inferred from the structure of an extracytoplasmic solute receptor protein. Mol Microbiol 57(1):143-155. doi:10.1111/j. 1365-2958.2005.04691.x

Nakayama K, Kelly SM, Curtiss R (1988) Construction of an Asd+ expression-cloning vector-stable maintenance and high-level expression of cloned genes in a Salmonella vaccine strain. Biotechnology 6:693-697

Newell DG, Fearnley C (2003) Sources of Campylobacter colonization in broiler chickens. Appl Environ Microbiol 69(8):43434351

Newell DG, Koopmans M, Verhoef L, Duizer E, Aidara-Kane A, Sprong H, Opsteegh M, Langelaar M, Threfall J, Scheutz F, van der Giessen J, Kruse H (2010) Food-borne diseases-the challenges of 20 years ago still persist while new ones continue to emerge. Int J Food Microbiol 139(Suppl 1):S3-S15. doi:10. 1016/j.ijfoodmicro.2010.01.021

Panthel K, Meinel KM, Sevil Domenech VE, Trulzsch K, Russmann H (2008) Salmonella type III-mediated heterologous antigen delivery: a versatile oral vaccination strategy to induce cellular immunity against infectious agents and tumors. Int $\mathrm{J}$ Med Microbiol 298(1-2):99-103. doi:10.1016/j.ijmm.2007.07.002

Parkhill J, Wren BW, Mungall K, Ketley JM, Churcher C, Basham D, Chillingworth T, Davies RM, Feltwell T, Holroyd S, Jagels K, Karlyshev AV, Moule S, Pallen MJ, Penn CW, Quail MA, Rajandream MA, Rutherford KM, van Vliet AH, Whitehead S, Barrell BG (2000) The genome sequence of the food-borne pathogen Campylobacter jejuni reveals hypervariable sequences. Nature 403(6770):665-668

Pawelec DP, Korsak D, Wyszynska AK, Rozynek E, Popowski J, Jagusztyn-Krynicka EK (2000) Genetic diversity of the Campylobacter genes coding immunodominant proteins. FEMS Microbiol Lett 185(1):43-49

Prokhorova TA, Nielsen PN, Petersen J, Kofoed T, Crawford JS, Morsczeck C, Boysen A, Schrotz-King P (2006) Novel surface polypeptides of Campylobacter jejuni as traveller's diarrhoea vaccine candidates discovered by proteomics. Vaccine 24(40-41):6446-6455. doi:10.1016/j.vaccine.2006.05.085

Sambrook J, Russel DW (2001) Molecular cloning-a laboratory manual, 3rd edn. Cold Spring Harbor Laboratory Press, Cold Spring Harbor, New Yok

Schoen C, Stritzker J, Goebel W, Pilgrim S (2004) Bacteria as DNA vaccine carriers for genetic immunization. Int J Med Microbiol 294(5):319-335

Schoen C, Loeffler DI, Frentzen A, Pilgrim S, Goebel W, Stritzker J (2008) Listeria monocytogenes as novel carrier system for the development of live vaccines. Int J Med Microbiol 298(1-2): 45-58. doi:10.1016/j.ijmm.2007.09.002

Schrotz-King P, Prokhorova TA, Nielsen PN, Crawford JS, Morsczeck C (2007) Campylobacter jejuni proteomics for new travellers' diarrhoea vaccines. Travel Med Infect Dis 5(2):106-109. doi:10.1016/j.tmaid.2006.01.019

Shi H, Wang S, Roland KL, Gunn BM, Curtiss R 3rd (2010) Immunogenicity of a live recombinant Salmonella enterica serovar Typhimurium vaccine expressing $p s p A$ in neonates and infant mice born from naive and immunized mothers. Clin Vaccine Immunol 17(3):363-371. doi:10.1128/CVI.00413-09

Shoaf-Sweeney KD, Larson CL, Tang X, Konkel ME (2008) Identification of Campylobacter jejuni proteins recognized by maternal antibodies of chickens. Appl Environ Microbiol 74(22):6867-6875. doi:10.1128/AEM.01097-08

Silva J, Leite D, Fernandes M, Mena C, Gibbs PA, Teixeira P (2011) Campylobacter spp. as a foodborne pathogen: a review. Front Microbiol 2:200. doi:10.3389/fmicb.2011.00200

Studier FW (2005) Protein production by auto-induction in high density shaking cultures. Protein Expr Purif 41(1):207-234

Torres-Escobar A, Juarez-Rodriguez MD, Gunn BM, Branger CG, Tinge SA, Curtiss R 3rd (2010) Fine-tuning synthesis of Yersinia pestis LcrV from runaway-like replication balanced-lethal plasmid in a Salmonella enterica serovar Typhimurium vaccine induces protection against a lethal $Y$. pestis challenge in mice. Infect Immun 78(6):2529-2543. doi:10.1128/IAI.00005-10

Wang S, Li Y, Scarpellini G, Kong W, Shi H, Baek CH, Gunn B, Wanda SY, Roland KL, Zhang X, Senechal-Willis P, Curtiss R 3rd (2010) Salmonella vaccine vectors displaying delayed antigen synthesis in vivo to enhance immunogenicity. Infect Immun 78(9):3969-3980. doi:10.1128/IAI.00444-10

Wang S, Kong Q, Curtiss R 3rd (2013) New technologies in developing recombinant attenuated Salmonella vaccine vectors. Microb Pathog 58:17-28. doi:10.1016/j.micpath.2012.10.006

Wyszynska A, Raczko A, Lis M, Jagusztyn-Krynicka EK (2004) Oral immunization of chickens with avirulent Salmonella vaccine strain carrying $C$. jejuni 72Dz/92 cjaA gene elicits specific humoral immune response associated with protection against challenge with wild-type Campylobacter. Vaccine 22(11):13791389. doi:10.1016/j.vaccine.2003.11.001

Wyszynska A, Zycka J, Godlewska R, Jagusztyn-Krynicka EK (2008) The Campylobacter jejuni/coli cjaA (cj0982c) gene encodes an $\mathrm{N}$-glycosylated lipoprotein localized in the inner membrane. Curr Microbiol 57(3):181-188. doi:10.1007/s00284-008-9171-3

Xin W, Wanda SY, Zhang X, Santander J, Scarpellini G, Ellis K, Alamuri P, Curtiss R 3rd (2012) The Asd+ -DadB+ dualplasmid system offers a novel means to deliver multiple protective antigens by a recombinant attenuated Salmonella vaccine. Infect Immun 80(10):3621-3633. doi:10.1128/IAI. 00620-12 